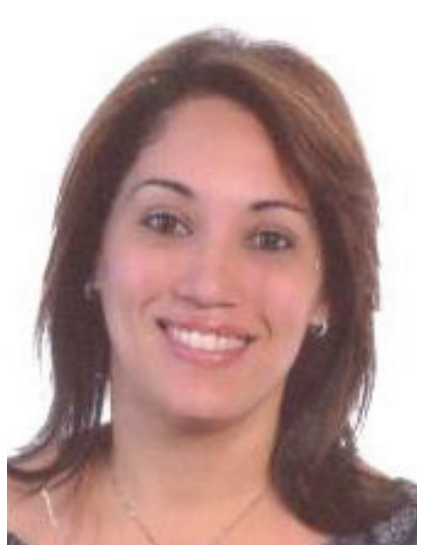

NOTAS BIBLIOGRÁFICAS

\title{
Reseña del libro Evaluación de habilidades y competencias en Educación Superior ${ }^{1}$
}

\author{
Rebeca Espinosa *
}

Este libro abarca diferentes temas relacionados con las mejores prácticas de evaluación de las competencias y las habilidades que deben desarrollar los estudiantes a lo largo de sus años de estudio en las instituciones de Educación Superior.

Actualmente, en el mundo académico se le está dando mucha importancia al proceso de evaluación del saber hacer, sin restarle prioridad al saber. Sin embargo, dado que las instituciones de Educación Superior están valorando cada vez más lo que se aprende en las asignaturas de práctica y en aquellas de contenido profesional, es imperativo saber cómo evaluar dichas competencias, es decir, qué y cómo aprenden los estudiantes. Por lo que, básicamente, el libro está enfocado a docentes de grado y postgrado de todas las disciplinas y de diferentes generaciones.

El libro está dividido en once capítulos y estos a su vez tienen diferentes subtemas. Para fines de esta reseña se presentarán los capítulos con sus títulos y las ideas centrales de cada uno de ellos.

\section{Importancia de un buen diseño de evaluación de las destrezas prácticas \\ En la actualidad, se prevé que los estudiantes demuestren unas destrezas y habilidades que pongan de manifiesto que no solo han llegado a dominar los contenidos teóricos de las asignaturas, sino también la habilidad de trasladar a la práctica lo aprendido en contextos de la vida real, es decir, que los estudiantes deben demostrar ser competentes; y ser conscientes de que se valora como importante tanto la capacidad de saber como la capacidad de hacer.}

Cuando los docentes llevan a cabo el proceso de evaluación deben plantearse las siguientes interrogantes: ¿Por qué estoy evaluando? ¿Qué fines concretos pretendo? ¿Qué estoy evaluando? ¿Cómo estoy evaluando? ¿Quién está en mejor situación para evaluar? y ¿Cuándo debe tener lugar la evaluación?

Así, se consideraquela evaluación necesariamente debe formar parte del proceso de aprendizaje, es decir, que no es un paso exclusivo de un momento determinado en el período académico, sino que se debe evaluar constantemente.

Por lo que, tomando en cuenta que desde el primer momento el docente y el estudiante saben lo que se desea lograr con el diseño de la evaluación y, luego, con la planificación detallada del contenido, es importante considerar las cuestiones enlazadas que subyacen en el diseño de la evaluación, tales como: ¿Qué evaluar (teoría, práctica, proceso, resultado)? ¿Por qué evaluar (retroalimentar, facilitar, calificar, revisión de valores, motivar)? ¿Quién debe evaluar (docente, compañeros, uno mismo, otros)? ¿Cómo evaluar (ensayos, portafolios, seminarios, pruebas de simulación, exámenes, tesis, otros)? ¿Cuándo evaluar (al final, a mitad del curso, al cabo de seis semanas)?

En ese mismo orden, se entiende que una evaluación eficaz de competencias debe ser válida, fiable, consistente, justa, inclusiva, controlable, auténtica, exigente, motivadora, eficiente. $Y$, en el mundo ideal, sería conveniente que fuera entretenida, elegante por su sencillez, fácil de calcular, carente de complicaciones, reducida en tiempo y sinóptica. En conclusión,

\footnotetext{
${ }^{1}$ Brown, S. \& Pickford, R. (2013). Evaluación de habilidades y competencias en Educación Superior. Madrid: Narcea

" Licenciada en Psicología Organizacional por la Pontificia Universidad Católica Madre y Maestra, Campus de Santiago, y magíster en Administración de Empresas y en Pedagogía Universitaria por esa misma universidad. Directora del Departamento de Psicología del Campus de Santiago.
}

Encuentre el texto en, http://www.pucmm.edu.do/STI/campus/CDP/ComuncacionPublicaciones/Paginas/CuadernodePedagogiaUniversitaria.aspx

Cuaderno de Pedagogía Universitaria Año 10/ N. 19 /enero-junio 2013 / Santiago, Rep. Dom.: PUCMM / p.26-31 
se debe expresar de manera precisa lo que se desea evaluar del estudiantado y esto puede ayudarlos a identificar sus propios objetivos de aprendizaje.

Finalmente, en el siguiente cuadro se presenta una selección adecuada de verbos que permite a los estudiantes recibir la evaluación cuando hayan alcanzado una confianza razonable en su rendimiento, en componentes diferentes de una tarea extensa.

\section{Lenguaje que se utiliza en los resultados del aprendizaje práctico}

Los verbos que se emplean para definir los resultados del aprendizaje práctico pueden ser muy útiles como primera etapa para caer en la cuenta acerca de lo que queremos juzgar y calificar. La siguiente lista está dispuesta en orden de complejidad de manera que los diseñadores de las tareas pueden ajustarla al nivel y complejidad correspondiente:

Demuestra, muestra, haz una lista, destaca, describe

Diseña, crea, desarrolla, implementa, haz, planea, construye, articula, resuelve

Identifica, discrimina entre, selecciona, ordena, escoge, prioriza

Explica, traduce, especifica, discute, razona, pon a prueba

Desarrolla, posibilita, facilita, diseña, analiza

Compara, contrasta, juzga, evalúa

\section{El valor de la evaluación formativa en la evaluación de las destrezas prácticas}

Cuando se van a evaluar las competencias que se desea desarrollen los estudiantes, hay que ser muy específicos con el tipo de actividad académica que se va a asignar, de manera que ellos puedan demostrar sus conocimientos y competencias y sepan la mejor forma de hacerlo.

De manera que, para lograr lo anterior, se requiere que el docente revise los procedimientos de evaluación y el diseño curricular en su conjunto y dejar de considerar los contenidos como lo más importante del proceso formativo.

Se plantea la importancia de la retroinformación en el proceso de la evaluación, como una buena estrategia, ya que tanto los comentarios como las sugerencias que los docentes realizan a los trabajos entregados por los estudiantes hacen que estos puedan mejorar su proceso de aprendizaje. Lamentablemente, algunos estudiantes solo se preocupan por la calificación y no le dan importancia a la retroinformación, porque no saben utilizarla de manera constructiva.

A continuación, algunas sugerencias al profesorado sobre el uso de la retroinformación:

- Dedicar tiempo explicando a los estudiantes la importancia de la retroinformación en el proceso de aprendizaje.

- Realizar la retroinformación de la manera más personal posible, ya sea individual o colectivamente.

- Devolver los trabajos a los estudiantes lo más rápido posible.
- Cuidar el lenguaje que va a utilizar para ofrecer la retroinformación, ya que se debe evitar la crítica destructiva de la persona.

- Considerar la posibilidad de dar oportunidades de entrega repetidamente, ya que en ocasiones el estudiante hace un mejor trabajo cuando se le ofrece una segunda oportunidad.

\section{Evaluar a estudiantes con necesidades educativas especiales}

La práctica inclusiva enriquece la experiencia de todas las personas que estudian y trabajan en la universidad; de manera que la práctica de la evaluación inclusiva debe abordarse de forma sistemática, lo que puede significar que el profesorado deba participar en sesiones de formación para la práctica de la evaluación. En ese sentido, se sugiere que se determinen las alternativas que se van a llevar a cabo en el momento del diseño curricular, de manera que los estudiantes con necesidades educativas especiales no se sientan marginados por recibir un trato diferente; para esto se sugiere implicar a esos estudiantes y a otros alumnos en el diseño varias alternativas de evaluación diferentes a las que conocen tradicionalmente.

Las autoras afirman que la inmensa mayoría de los estudiantes que presentan necesidades educativas especiales en las instituciones de educación superior es disléxica (mala o lenta caligrafía, lectura lenta, dificultades estructurales y gramaticales, problemas con la memoria a corto plazo, desorganización). En otros casos, los estudiantes pueden presentar dificultades visuales, lo cual representa graves inconvenientes, pues la educación superior depende en gran medida de la lectura y la escritura como elementos clave de las transacciones académicas. Otra situación la representan los estudiantes con problemas auditivos, cuya primera lengua es la de signos, pudieran estar en inferioridad de condiciones por su falta de familiaridad con los vocabularios y su menor soltura al expresarse por escrito. Otra población discapacitada la representan los estudiantes con ciertos problemas de salud mental, que pueden llevar a los individuos a la incapacidad para abordar la organización de un trabajo, dificultades en la aplicación y problemas de asistencia.

A continuación se presentan algunos consejos para el diseño de un enfoque inclusivo de evaluación de las competencias:

- Incluir varias alternativas en los planes de evaluación en la fase de diseño de la asignatura, en lugar de esperar que se presenten las situaciones de estudiantes con necesidades educativas especiales.

- Realizar un análisis de necesidades en relación a los requisitos de la evaluación si se sabe de antemano que se tiene ese tipo de estudiantes.

- Trabajar con profesionales expertos sobre el tema y crear una base de conocimientos sobre las necesidades de esos estudiantes.

- Recibir retroinformación de los estudiantes discapacitados en relación a la práctica de evaluación inclusiva.

- Tomar en cuenta los requisitos de accesibilidad y seguridad de los estudiantes discapacitados que deben participar en prácticas y salidas para el estudio. 
Finalmente, las autoras promueven que hay algunas universidades que han creado grupos de asesores de estudiantes discapacitados para que trabajen con los docentes con la finalidad de promover prácticas pedagógicas inclusivas.

\section{Evaluar a estudiantes "diferentes"}

En este apartado, las autoras hacen referencia a la promoción de un trato igualitario a los estudiantes, es decir, sin ningún tipo de discriminación, por lo que el docente debe estar alerta para actuar con justicia y ofrecer a cada estudiante una experiencia de aprendizaje equivalente.

Se propone además, que de las competencias que debe desarrollar el estudiante de Educación Superior, está el trabajo en equipo; de manera que, es labor del docente evitar las minorías que se crean dentro del grupo de clases cuando se asignan trabajos en conjunto y aprovechar la riqueza que supone el tener estudiantes extranjeros. Se propone además, integrar al diseño curricular la sensibilidad intercultural, para garantizar que los estudiantes no se sientan discriminados por las exigencias de alguna tarea en particular.

Algunas sugerencias para garantizar la equivalencia de la experiencia para los estudiantes de diferentes culturas al evaluar las destrezas, son: especificar lo que se espera en relación a la originalidad, utilizar estudios de casos de diversos contextos nacionales y culturales, evitar referirse a un determinado grupo de edad al hacer referencias culturales y/o sociales que puedan marginar a los más jóvenes o mayores, ser prudente antes de dar por supuesto una norma como universal, tomar en cuenta el lenguaje utilizado para ofrecer retroinformación, y, finalmente, centrarse siempre en los resultados en lugar de la persona.

5. Promover habilidades y destrezas para el aprendizaje autónomo Las autoras afirman que las destrezas de aprendizaje autónomo consolidadas han sido una característica habitual entre los estudiantes que ingresaban en la educación superior. Sin embargo, plantean que muchos de los estudiantes que llegan a la universidad no tienen las destrezas de estudio necesarias para salir airosos.

Tomando como referencia lo que se plantea en el Modelo Educativo de la PUCMM (2011) donde se plantea que "el estudiante asume una serie de compromisos con el fin de lograr aprendizajes significativos para sí mismo y para su grupo", se entiende que es labor del docente ser "facilitador y promotor del aprendizaje de los estudiantes". De manera que, se les recomienda tener en cuenta algunas consideraciones para desarrollar el aprendizaje autónomo en el estudiantado, estas son:

- Investigar diversas formas en que los estudiantes puedan demostrar sus destrezas de aprendizaje autónomo, se pueden incluir los diarios de clase, los portafolios, las reflexiones, entre otros.

- Utilizar los criterios de evaluación para aclarar lo que desea que logren los estudiantes, es decir, hacer que los criterios y las ponderaciones reflejen las actividades y enfoques que se desean privilegiar.
- Incluir la autoevaluación en las entregas de trabajos, pues las autoras consideran que es un elemento eficaz para determinar las áreas de mejora del estudiante.

- Incluir las estrategias de aprendizaje basado en proyectos y aprendizaje basado en problemas, ya que según las autoras, esto ayuda a concentrarse más en el proceso que en el producto de aprendizaje.

\section{Evaluar las presentaciones orales y las disertaciones}

En este apartado se recomienda establecer con precisión por qué se va a evaluar la destreza de oratoria y qué se va a evaluar, esto debe variar dependiendo de las asignaturas, las disciplinas y el nivel al que se desea desarrollar la competencia de comunicación oral.

Es importante no limitar la evaluación de las destrezas oratorias de los estudiantes a la disertación formal unidireccional y cara a cara; ya que la experiencia oral del estudiante puede enriquecerse mediante diversos formatos de evaluación. Algunos de los formatos más interactivos son los seminarios, las exposiciones orales, los debates, entre otros, y permiten que los docentes evalúen al estudiante en cuanto al nivel de aprendizaje y desarrollo de destrezas de comunicación, que si sólo lo hiciera con las disertaciones tradicionales.

Lo más importante cuando se evalúa la competencia de comunicación oral es contar con los criterios adecuados de evaluación. Se debe tomar en cuenta el contenido y las calificaciones correspondientes a cada criterio. A partir de unos criterios claros de evaluación, se debe ser capaz de seleccionar la manera más adecuada de evaluación e incluso analizar si es conveniente involucrar a terceros en el proceso evaluativo.

En relación a esto último, las autoras sugieren que se deben establecer criterios en relación a quienes van a ser los responsables de evaluar las presentaciones orales. En ese sentido, plantean varias opciones, una de ellas es la autoevaluación, la cual se puede realizar a través de videos que realicen los estudiantes sobre su exposición de manera que les sirve de crítica constructiva para desarrollar sus propias reflexiones; otros actores implicados en el proceso, pueden ser los profesores y expertos externos; los compañeros de curso, para quienes se considera una buena experiencia ya que podrían mejorar sus propias habilidades de oratoria. Finalmente, las presentaciones orales en equipo, la cual es excelente porque reduce la cantidad de trabajos que el docente debe calificar y por otro lado, al calificar a los estudiantes como equipo hace que todos tengan un interés personal en mejorar la actuaciones de los demás miembros del grupo, ya que se preparan mejor y hacen que los demás se centren en la exposición y no en las personalidades de los oradores. En definitiva, lo importante es tener claridad de lo que se va a evaluar.

\section{Evaluar las competencias prácticas en los trabajos de laboratorio} Existen diferentes enfoques, algunos tradicionales y otros nuevos, que sirven para evaluar las destrezas prácticas en todo tipo de laboratorios. En la Tabla 1 (adaptada de Hughes, 2004) se presentan diversas estrategias de evaluación. 
Tabla 1. Evaluación de los informes de laboratorio

\begin{tabular}{|c|c|c|}
\hline Estrategias & Ventajas & Inconvenientes \\
\hline Los estudiantes trabajan en equipo. & $\begin{array}{l}\text { Oportunidades de enseñanza a cargo de los } \\
\text { compañeros y de mejora del aprendizaje. } \\
\text { Reduce la carga de trabajo de la corrección. }\end{array}$ & $\begin{array}{l}\text { No aborda el problema de los estudiantes que no } \\
\text { utilizan suficientemente la retroinformación del } \\
\text { tutor. }\end{array}$ \\
\hline $\begin{array}{l}\text { Calificar solo una muestra o selección de } \\
\text { los ejercicios de laboratorio. }\end{array}$ & Reduce la carga de trabajo de la corrección. & $\begin{array}{l}\text { Si los estudiantes saben que no se calificarán los } \\
\text { informes, puede que no pongan tanto interés } \\
\text { en ellos. No aborda la infrautilización de la } \\
\text { retroinformación. }\end{array}$ \\
\hline $\begin{array}{l}\text { La calificación corre a cargo de estudiantes } \\
\text { de postgrado. }\end{array}$ & $\begin{array}{l}\text { Reduce la carga de trabajo de corrección de } \\
\text { los profesores. }\end{array}$ & $\begin{array}{l}\text { Puede haber incongruencia en las } \\
\text { calificaciones. No aborda la infrautilización de la } \\
\text { retroinformación. }\end{array}$ \\
\hline $\begin{array}{l}\text { Pedir a los estudiantes que entreguen sus } \\
\text { informes en un formato especificado. }\end{array}$ & Reduce el tiempo de corrección. & $\begin{array}{l}\text { Reducen la iniciativa del estudiante. El formato } \\
\text { relleno puede pasarse con facilidad de un grupo } \\
\text { a otro. }\end{array}$ \\
\hline Utilizar preguntas de opciones múltiples. & $\begin{array}{l}\text { Pueden evaluarse por ordenador, } \\
\text { eliminando la carga de trabajo de la } \\
\text { corrección. Puede darse retroinformación } \\
\text { inmediata [En la universidad se han } \\
\text { realizado este tipo de evaluaciones en } \\
\text { Medicina, aprovechando eluso del Moodle- } \\
\text { Plataforma Virtual de Aprendizaje]. }\end{array}$ & $\begin{array}{l}\text { No se desarrollan las destrezas de redacción de } \\
\text { informes }\end{array}$ \\
\hline Evaluación de los informes por compañeros. & $\begin{array}{l}\text { Reduce significativamente la carga de } \\
\text { trabajo de corrección del profesorado. }\end{array}$ & $\begin{array}{l}\text { Requiere orientaciones claras y explícitas de } \\
\text { calificación. }\end{array}$ \\
\hline
\end{tabular}

Cada una de esas estrategias puede ser eficaz para reducir la carga que supone la evaluación de los informes de laboratorio; además, algunas de ellas facilitan los momentos adecuados para la evaluación formativa y la mejora del aprendizaje de los estudiantes.
Por otro lado, existen varios métodos para evaluar las destrezas prácticas de laboratorio, las cuales se presentan en la Tabla 2:

Tabla 2. Comparación de distintos métodos para evaluar las destrezas prácticas de laboratorio

\begin{tabular}{|l|l|}
\hline Método de Evaluación & Características \\
\hline $\begin{array}{l}\text { Informe tradicional } \\
\text { de laboratorio (por } \\
\text { escrito) }\end{array}$ & $\begin{array}{l}\text { Facilita a los estudiantes una práctica frecuente de redactar informes de laboratorio. Introduce en una forma } \\
\text { disciplinada de enfocar la medida y el registro de los resultados. Proporciona un informe detallado. Puede facilitar a } \\
\text { los estudiantes una información regular sobre sus actividades. Se corre el riesgo de que se copien los resultados. Para } \\
\text { el profesorado, la corrección y la calificación supone una carga pesada. }\end{array}$ \\
\hline Diario de laboratorio & $\begin{array}{l}\text { Se redacta durante la sesión de laboratorio, garantizando la integridad de los datos y que sea obra de cada estudiante. } \\
\text { Puede resultar pesado para el estudiante y para el profesorado. Las versiones modernas exigen que los estudiantes } \\
\text { completen sus diarios en PC o portátil en el mismo laboratorio y que lo envíen electrónicamente. }\end{array}$ \\
\hline $\begin{array}{l}\text { Examen clínico objetivo } \\
\text { y estructurado (ECOE) }\end{array}$ & $\begin{array}{l}\text { Los estudiantes rotan por una seria de puestos en los que se les pide que lleven a cabo diversos procedimientos. Exige } \\
\text { una planificación detallada. }\end{array}$ \\
\hline $\begin{array}{l}\text { Observación de } \\
\text { estudiantes }\end{array}$ & $\begin{array}{l}\text { Puede ser exhaustiva, pero exige tiempo y dedicación intensiva del profesorado. Los estudiantes pueden comportarse } \\
\text { de forma diferentes cuando el tutor no los está mirando. }\end{array}$ \\
\hline $\begin{array}{l}\text { Ejemplificación de una } \\
\text { destreza práctica }\end{array}$ & Es una forma rápida y fiable de evaluación y de retroinformación, pero lleva tiempo. \\
\hline $\begin{array}{l}\text { Presentación y defensa } \\
\text { oral de los resultados }\end{array}$ & $\begin{array}{l}\text { Combate el plagio. Puede dar mucha información, es útil averiguar si el estudiante ha comprendido la tarea realizada. } \\
\text { En el momento de la evaluación oral, se trasmite inmediatamente la información al estudiante. Lleva tiempo y su } \\
\text { organización puede resultar problemática. }\end{array}$ \\
\hline Ejercicios de simulación & Respaldalaprácticay puedeser razonablementeauténtica, aunquenodel todo.Suorganización puederequerirtiempoy recursos. \\
\hline
\end{tabular}


Además de las presentadas anteriormente, existe otra modalidad de evaluación de prácticas que es el aula multimedia, la cual encierra un potencial enorme para unas evaluaciones ricas, interactivas e interesantes. Su uso más común es para pruebas digitales de opciones múltiples u otra clase de pruebas similares [verdadero o falso, pareo, respuestas cortas, otros] y ofrece la oportunidad de que el estudiante compruebe por qué son correctas o incorrectas sus respuestas, además, alivian el trabajo de corrección del profesor. Una consideración importante es que el banco de preguntas puede exigir tiempo, dedicación y cuidado. Para que estas evaluaciones se puedan llevar a cabo se requiere de un equipo multidisciplinar de expertos en contenido, expertos pedagógicos y diseñadores con buenas capacidades tecnológicas. Las desventajas de esta modalidad son los posibles fallos de las TIC y problemas de seguridad con los hackers.

\section{Evaluar los productos y los artefactos creados en un estudio o en un taller}

La evaluación de artefactos o producciones son fundamentales en muchas asignaturas de carácter técnico. En algunos casos es necesario evaluar la precisión técnica, la creatividad, la originalidad o el proceso de producción. A continuación se presentan algunas sugerencias importantes para la evaluación de la producción de artefactos o producciones diversas:

- $\quad$ Especificar el nivel de logro deseado.

- Determinar el nivel adecuado de desarrollo de las competencias [técnicas] en el programa de aprendizaje.

- Facilitar las informaciones y expectativas del programa desde el inicio, puede ser con ejemplos prácticos.

- Centrar la retroinformación formativa y las críticas en el trabajo, no en la persona.

- Cuando el artefacto sea de uso comercial, puede apoyarse en la evaluación de externos (usuario final o cliente).

- Solicitar evidencia al estudiante del uso de la originalidad y creatividad (cuando sea el caso) sobre el proceso creativo completo.

\section{Evaluar interpretaciones y representaciones "en vivo"}

La interpretación "en vivo" es esencialmente efímera y como tal es una de las áreas más difíciles de evaluar con justicia. Al diseñar una asignatura de carácter interpretativo hay que buscar el equilibrio entre los elementos prácticos y teóricos del programa de estudios. Los trabajos escritos y las actuaciones han de ser complementarios, de manera que el trabajo intelectual respalde la actividad.

Para un proceso justo de evaluación se recomienda: animar a los estudiantes a ver sus propias actuaciones en video y eso les permite hacer juicios realistas de su trabajo, ayudar a los estudiantes a que se centren en los medios potenciales para mejorar y descubrir técnicas que precisen más ensayos, trabajar con otros evaluadores para determinar el umbral de rendimiento, evitar el efecto halo, tomar en cuenta los logros grupales e individuales para lo cual debe establecer los criterios de evaluación, entre otros.

\section{Evaluar los procesos}

En la actualidad, el enfoque de la Educación Superior está centrado en lo que el estudiante aprende. Las instituciones modernas han desarrollo sus modelos por competencia de acuerdo a su contexto. $Y$ para ser realmente eficaces en este modelo, hay que demostrar que estas competencias no están separadas del conocimiento de las asignaturas sino que lo incluyen y que se debe procurar su aplicación en contextos reales.

Cuando los docentes evalúen los procesos, deben considerar qué ventajas tiene para el estudiante y para la sociedad, sobre los resultados. Es decir, qué se debe tener si lo que se va a evaluar es el resultado o el proceso. En ese mismo orden, se propone centrarse más en el proceso que en el producto, como una medida para reducir el plagio, para lo cual los docentes deben asegurarse de que el trabajo sea realmente del estudiante.

Existen varios enfoques posibles para evaluar los procesos, los cuales se presentan a continuación:

- Exámenes: se utilizan para evaluar destrezas prácticas. Se consideran fáciles de administrar y de controlar. Quienes están en contra de los exámenes apuntan a que siguen existiendo trampas, que se realizan bajo un ambiente de presión y que perjudica a algunos estudiantes (disléxicos, por ejemplo). Para un adecuado uso de estos, se recomienda un adecuado diseño de preguntas y determinar qué se desea conseguir al decidir el número y el tipo de preguntas.

- Ejercicios de simulación de actividades profesionales: sirven para evaluar la capacidad de los estudiantes para priorizar y trabajar con documentación desconocida, emulando situaciones de trabajo del mundo real. La carpeta de preparación de documentos puede requerir mucho trabajo, pero pueden continuar utilizándose en cursos posteriores.

- Exámenes en libro abierto: son útiles cuando se evalúa el manejo de la información por parte de los estudiantes, en vez del recuerdo de la misma. Para que sea efectivo, se debe orientar a los estudiantes acerca del concepto de los mismos para que no se limiten a copiar textos.

- Hojas de examen para llevar: pueden utilizarse cuando el objetivo es demostrar la aplicación de conocimientos y no su recuerdo. Las preguntas deben formularse cuidadosamente para centrarse en la demostración del dominio de destrezas. Una desventaja es que no hay garantía de que el trabajo sea fruto del estudiante, ya que se realiza fuera del aula. 
- Exámenes orales: sirven para evaluar destrezas prácticas y dan la oportunidad de poner a prueba a los estudiantes uno a uno. Sin embargo, si los estudiantes vienen de un contexto cultural donde se limita la interacción cara a cara, estos pueden ser perjudicados.

- La observación: sirve para evaluar destrezas y es útil por su autenticidad; sin embargo, exige mucho tiempo del observador sobre todo si el grupo de estudiantes es muy grande.

- La reflexión personal: es un ejercicio formativo muy útil donde el estudiante puede valorar los problemas encontrados en el transcurso de un proyecto. El docente debe acompañar al estudiante a desarrollar comentarios reflexivos significativos. seguimiento tanto del docente como del supervisor de práctica.

Con todo lo anteriormente debidamente explicitado, se deben desarrollar algunas actividades adicionales encaminadas a mantener la consistencia de las prácticas, tales como: realizar un manual de prácticas, organizar sesiones de formación de evaluadores, hacer visitas periódicas, otras.

Un factor que se debe considerar en relación a las prácticas son los imprevistos que se puedan presentar, tales como: cierre del centro de trabajo, despido de la persona, acoso, maltrato o cualquier conducta indeseable, una emergencia (desastre natural, por ej.), entre otros. En cualquiera de estos casos, las autoras recomiendan que se negocie con el estudiante implicado algunas alternativas que puedan salvar la situación, como puede ser la reubicación del centro de trabajo.

- La evaluación por compañeros: es un enfoque útil para los evaluadores porque les ayuda a mejorar destrezas similares y a aprender de las experiencias de otros. Para que sea realmente justa, se recomienda establecer unos criterios explícitos.

- La evaluación intragrupo: es conveniente cuando se trabaja en grupo pedirle a los demás compañeros que evalúen las contribuciones realizadas por los demás miembros. Como en otros enfoques, es importante determinar los criterios de evaluación para que no haya sesgos inconscientes.

- La exposición: es una forma rápida de dar a conocer a los estudiantes un amplio conjunto de enfoques. Además es una oportunidad para que los estudiantes comenten este proceso con diversos evaluadores.
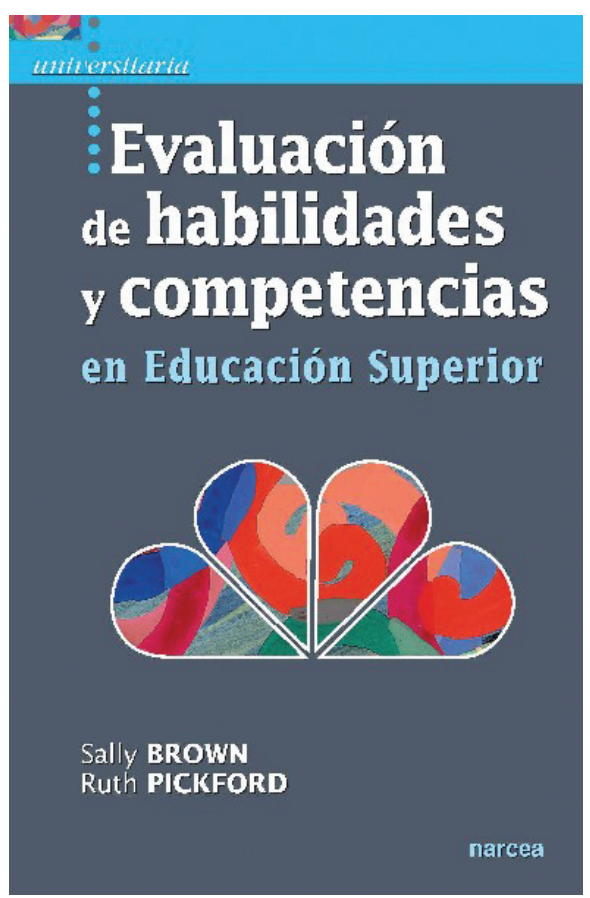

En muchas disciplinas [por ej. Medicina y Psicología en PUCMM], los estudiantes tienen la oportunidad de prestar sus servicios a la comunidad. En estos casos, las autoras plantean la posibilidad de que los clientes/ usuarios participen en el proceso de evaluación de las prácticas.

Resumiendo, se puede afirmar que este libro es de utilidad para los docentes de Educación Superior, ya que para dar continuidad a los lineamientos del Modelo Educativo de la PUCMM, el profesorado debe centrarse en "el estudiante y en su proceso de aprendizaje, atendiendo a todas las dimensiones de la persona, de forma que alcance la integración de conocimientos, destrezas, habilidades, valores actitudes en todos los ámbitos de su vida" y precisamente con este libro podemos obtener diversas estrategias para evaluar las competencias y destrezas que

- La triangulación: cuando se evalúa algo tan complejo como el proceso, este enfoque es particularmente el más deseable. Es decir, que la evaluación depende del estudiante, los compañeros y el profesor.

\section{Evaluar a estudiantes en práctica}

La mayoría de las disciplinas universitarias realizan prácticas en diferentes tipos de instituciones, estas experiencias de aprendizaje implican evaluadores diferentes al docente de la asignatura. De manera que, se recomienda que los tutores de prácticas realicen una planificación que garantice una adecuada experiencia de aprendizaje y que las evaluaciones de los estudiantes sean lo más justas posible.

Con la finalidad de proporcionar una experiencia de aprendizaje significativa en las prácticas, es importante que se detallen los resultados esperados, se haga una lista de las competencias que se desean desarrollar, un cronograma de actividades, un protocolo de deben desarrollar los estudiantes; lo cual nos lleva a ser creativos en la manera en que vamos a desarrollar el proceso de enseñanzaaprendizaje desde las asignaturas que impartimos.

Además, se debe puntualizar que la evaluación bien planificada, diseñada y ejecutada ofrece una excelente oportunidad para enriquecer el proceso de enseñanza-aprendizaje y puede repercutir positivamente en el rendimiento de los estudiantes.

Finalmente, tal como se plantea en el Modelo Educativo de la PUCMM (2011), "se necesita la implementación de diversas formas innovadoras de evaluación que permitan al estudiante una reflexión constante sobre sus avances en el aprendizaje y que contribuyan a su formación, refuercen su autoestima y su motivación para un aprendizaje a lo largo de la vida". 